\title{
Las TICs como herramienta pedagógica para el fortalecimiento de las relaciones interpersonales y la sana convivencia ${ }^{1}$
}

\section{Tics as a pedagogical tool for the strengthening of interpersonal relationships and healthy coexistence}

DOI: http://dx.doi.org/10.17981/cultedusoc.9.3.2018.69

Artículo de investigación. Fecha de recepción: 15/06/2018. Fecha de aceptación: 27/11/2018

Elcie Támara-Camacho ${ }^{2}$;

Norma Palacin-Moya; Lorenza Jiménez -Ortega; Margarita De La Cruz -Muñoz; Esperanza Palacin-Moya; Sofía Martínez-Rambal; Arturo Martinez-Isaza; Jaime Meriño-Farías; Sahilly Ortega-Muñoz; Luz Ortega-Muñoz;

Mary Camacho-Támara; Abnovis De aguas-Barraza; Oscar Salas-Contreras; Sirith Martinez-Rambal; Juan Puerta-Pacheco; Nurys Barros-Vargas; Oladys Palacin-De Aguas; Roicy Támara-Camacho; Carmen Moya-Támara; Luis Pacheco-Ariza; Víctor Gutierrez-Castro; Milagro Tamara-Movilla;

Alma Camacho-Tamara y Nolida Carranza-Cáceres ${ }^{3}$

IED Santa Cruz de Bálsamo. Municipio de Concordia Magdalena (Colombia) elcietamara@hotmail.com

Para citar este artículo:

Tamara-Camacho, E., Palacin-Moya, N., Jiménez -Ortega, L., De La Cruz -Muñoz, M., Palacin-Moya, E., Martínez-Rambal, S., MartinezIsaza, A., Meriño-Farías, J., Ortega-Muñoz, S., Ortega-Muñoz, L., Camacho-Támara, M., De aguas-Barraza, A., Salas-Contreras, O., Martinez-Rambal, S., Puerta-Pacheco, J., Barros-Vargas, N., Palacin-De Aguas, O, Támara-Camacho, R., Moya-Támara, C., PachecoAriza, L., Gutierrez-Castro, V., Tamara-Movilla, M., Camacho-Tamara, A. y Carranza-Cáceres, N. (2018). Las TICs como herramienta pedagógica para el fortalecimiento de las relaciones interpersonales y la sana convivencia. Cultura. Educación y Sociedad 9(3), 581-590. DOI: http://dx.doi.org/10.17981/cultedusoc.9.3.2018.69

\section{Resumen}

Las relaciones interpersonales son la esencia de la convivencia. Actualmente resulta uno de los objetos de mayor interés al interior de los colegios. Con base a lo anterior se desarrolla el presente estudio que tiene como objetivo fortalecer las relaciones interpersonales y la sana convivencia en los estudiantes de la Institución Educativa Departamental Santa Cruz DE Bálsamo apoyados en las TIC como herramienta pedagógica. La metodología empleada fue desde la investigación cualitativa, con un alcance descriptivo, se tomó como muestra (51) estudiantes de los grados quinto y sexto. Dentro de los hallazgos encontrados, se identifican diversos problemas de convivencia y falta de comunicación asertiva, basados principalmente en la poca aceptación a las formas de pensar y actuar delos estudiantes, así como también frecuentes problemas de bullying.

Palabras clave: Relaciones interpersonales, convivencia, tics.

\section{Abstract}

Interpersonal relationships are the essence of coexistence. Currently it is one of the objects of greatest interest inside schools. Based on the above, the present study is developed to strengthen interpersonal relationships and healthy coexistence in the students of the Departmental Educational Institution Santa Cruz DE Bálsamo supported by ICT as a pedagogical tool. The methodology used was from qualitative research, with a descriptive scope, was taken as sample 51 students of the fifth and sixth grades. Within the found findings, diverse problems of coexistence and lack of assertive communication are identified, mainly based on the low acceptance to the ways of thinking and acting of the students, as well as frequent problems of bullying

Keywords: Interpersonal relationships, coexistence, tics.

\footnotetext{
1 Este artículo ha sido derivado del Programa de Fortalecimiento de la Cultura Ciudadana y Democrática CT+I a través de la IEP apoyada en TIC en el Departamento de Magdalena: CICLON.

2 Líder del grupo de investigación "Formadores con las TIC".

3 Docentes pertenecientes al grupo de investigación "Formadores con las TIC" de la IED Santa Cruz de Bálsamo. Municipio de Concordia Magdalena.
}

- The author; licensee Universidad de la Costa - CUC.

Cultura, Educación y Sociedad vol. 9 no. 3, pp. 581-590. Diciembre, 2018

Barranquilla. ISSN 2389-7724 Online 


\section{Introducción}

Las buenas relaciones interpersonales se hacen evidentes en un clima afectivo optimo, palpables en la calidad humana del ser y en el ambiente que genera este dónde se desenvuelve. Sin embargo, las carencias de ciertos valores humanos afectan la conducta de docentes, rector y estudiantes reflejándose en el desagrado por la actividad académica y específicamente en la escasa participación de compromisos de aula o extracurricular.

Este proyecto pretende hacer énfasis en el fortalecimiento de los valores humanos trabajados desde el aula y tomando como ejes la investigación y la tecnología de punta para así contrarrestar la crisis moral que aqueja a las nuevas generaciones y al mundo. Por lo tanto, tiene como objetivo primordial diseñar una herramienta pedagógica apoyada en TIC para fortalecer las relaciones Interpersonales y una sana convivencia en la IED Santa Cruz de Bálsamo.

Los valores dignifican y acompañan la existencia del ser, fluyen espontáneamente y se aprecian en las respuestas y reacciones frente a la cotidianidad. La IED representada a través de sus directivos, docentes y estudiantes requiere de un compromiso serio y constan te que contrarresta los brotes de intolerancia a que conlleva la modernidad y la transformación de la sociedad, esta acción debe estar abocada a la búsqueda de un número importante de instrumentos y estrategias que garanticen vivir mejor (González, Moreno, \& Rodríguez, 2015).

Lo anterior en el entendido de que la humanidad debe ser formada como sociedad de líderes éticos e integrales que puedan emplear habilidades para consensuar entre miembros de grupos y dirigir acciones por y para un bien común de países, organismos, instituciones entre otros (Kadi \& Acevedo, 2014)
Cada persona encuentra el potencial desarrollo de su vida y alcanza la plenitud en el encuentro con el otro, y por eso esta netamente envuelto en todas las relaciones naturales y sociales. Son el vínculo, en donde el individuo encuentra la posibilidad de satisfacción de sus necesidades, el logro de sus objetivos y el desarrollo de sus potencialidades. Por lo anterior las relaciones interpersonales llegan a considerarse según Clemente (2015), "un aspecto vital y de primera importancia para la existencia".

\section{Relaciones Interpersonales}

Las relaciones interpersonales resultan ser el intercambio que se da con los otros, las cuales toman en cuenta las emociones, sentimientos, intereses, actividades, que buscan siempre la cooperación y colaboración por parte de sus miembros,

"las relaciones interpersonales tienen lugar en una gran variedad de contextos, como la familia, los grupos de amigos, el matrimonio, los entornos laborales, los clubes sociales y deportivos, las comunidades religiosas y todo tipo de contextos donde existan dos o más personas en comunicación" (Mendez y Ryszard, 2005).

Son las relaciones interpersonales una necesidad propia para los individuos, por lo tanto, son diversos los factores que aquí influyen y que juegan un papel crucial en el desarrollo de los seres humanos, tales como la personalidad, las funciones cognitivas, el deseo y el estado afectivo.

Es posible que existan situación de tensión o disfuncionalidad en los sistemas sociales, y por ende se genere tensión y disfuncionalidad entre los miembros, es decir momentos de crisis e inestabilidad y para esto se debe contar con mecanismos que permitan la autorregulación, pero la realidad es que en sociedades como en la 
que vivimos ciertas veces las fluctuaciones son tan grandes que el sistema no es capaz de ajustarse y la retroalimentación positiva entra en acción, pero de forma ineficiente (Herrera, Certain y Calderón. 2014). Por tal razón la presente investigación busca el fortalecimiento de las relaciones interpersonales desde el sector educativo.

Es la personalidad la que se crea en el seno de las relaciones, y solo puede ser modificada en el seno de la misma. (Herriko. S.f).El producto del contacto y de la interacción entre las personas, de diferentes culturas depende, de muchos factores tanto de naturaleza personal, como contextual. No obstante, la influencia del contexto sin quietarle su nivel de importancia, parece estar subordinada a las actitudes y habilidades de cada individuo (Mendez y Ryszard, 2005).

Cuando se habla de actitudes, es importante hacer inferencia a lo que expresa Mendez y Ryszard, (2005), citando a Brislin (s.f),

"reacciones de la gente hacia el concepto, o usando el lenguaje coloquial sus sentimientos, creencias, y disposiciones hacia la acción. El concepto puede ser la persona, grupo, evento, objeto o una abstracción”. En las palabras de Allport "es una disposición psíquica y nerviosa, organizada por la experiencia que ejerce una influencia orientadora y dinámica, sobre las relaciones del individuo frente a todos los objetos y síntomas con los que está relacionado".

Se habla entonces también del aprendizaje de habilidades, las cuales facilitan al individuo un positivo desarrollo de las relaciones interpersonales, durante sus experiencias. Mendez y Ryszard, (2005), citando a Dorsch, definen el termino de habilidad como "la capacidad de realizar determinadas tareas, o resolver determinados problemas", subrayando su valor pragmático. Todas estas experiencias son adquirirdas por la experiencia y el entrenamiento, pero también dependen de la forma en la que la persona hace valor a sus derechos y necesidades.

Respecto a la relación del instructor con sus aprendices se puede señalar que tradicionalmente los maestros son el factor crucial de la educación en el aula; pues a través de su práctica pedagógica pueden generar una atmosfera, ordenada y orientada al aprendizaje.

Ascosh (1999) señala,

"que lo que caracteriza la actuación de los docentes es la tensión, la inseguridad y la agresividad igualmente. Montes \& Soriano (2000) reportan en sus observaciones en el aula de clases, comportamiento de estudiantes violentos. Así mismo agregan que el autoritarismo del docente en las aulas de clases impulsa a los estudiantes a proceder con agresión y hostilidad, y esto a su vez, genera un clima socio-emocional desfavorable para el adecuado desarrollo de los procesos de enseñanza y aprendizaje. De todo esto concluye, que a un no existe concordancia entre la realidad de las escuelas y lo planteado en el currículo".

De acuerdo a la investigación realizada por Rohner y Carrasco (2014), tiene en cuenta diversas interpretaciones que toman explícitamente las relaciones interpersonales en las instituciones educativas, lo relaciona a su vez con el clima que los estudiantes crean y como se da la convivencia siendo el docente el que direcciona estos escenarios.

Cuando se habla de las relaciones entre estudiantes y docentes, no se puede pensar que todo es armonía, pues se estima que son los estudiantes a los que se le dificulta llevar comportamientos asertivos, basados en la tolerancia y el respeto, e incluso el docente juega un papel fundamental, en el que dichas relaciones directas con él también se ven afectadas (Rohner y Carrasco 2014) 
Hay conductas que son universales y que determinan como se están llevando a cabo las relaciones con los otros, estas están manifestadas por amor, enojo, rabia, alegría, sonrisas, llantos, etc.

Se puede inferir entonces que la autoestima y las relaciones interpersonales son factores que van de la mano, de manera que el hombre fracasará en sus relaciones sino puede tener buenas relaciones consigo mismo, si no se respeta, sino se conoce, ni se acepta así mismo.

Por otra parte, el uso de las TIC a través de objetos virtuales de aprendizaje toma especial importancia, y entender la noción en el desarrollo de su concepto dentro del contexto social es un tema que resulta de interés (Suárez, 2016) (Betancurt et al., 2014) (Romero, Suárez y Rodríguez, 2018)

\section{Relaciones interpersonales en la escuela}

Es la escuela, uno de los escenarios donde se potencian las relaciones, pues en el contacto directo profesor - estudiante, se comparten sentimientos, valores, pensamientos, ideas etc. En las relaciones con personas del mismo género al interior de las Instituciones educativas se puede evidenciar respeto entre sí, los canales de comunicación son más asertivos, por lo tanto, cuando se presentan dificultades se intenta ayudar y cooperar para que todos sean escuchados, atendidos y valorados. Sin embargo, el conflicto siempre está, pueda que sea de menor grado.

\section{Convivencia escolar}

Se puede definir la convivencia como el clima de interrelaciones que se produce en la institución escolar, y que comprende las relaciones entre todos los miembros de la comunidad educativa (estudiantes, profesores/as, personal administrativo y padres de familia).
Según Jacques Delors en un informe presentado en la UNESCO en (1997) estableció que uno de los cuatro pilares de la educación es el aprender a vivir juntos; allí resalta la importancia de la convivencia en el aula para la educación además de los aspectos cognoscitivos e intelectuales.

Últimamente los medios de comunicación nos han dado a conocer la ocurrencia de problemas de convivencia en las escuelas con casos de violencia, acoso y mal trato de la que son objeto los estudiantes y hasta algunos maestros o docentes, nos pone de manifiesto el tipo de relaciones interpersonales que se vive en ella y también pone en tela de juicio la calidad de nuestro sistema educativo. La presencia de estos hechos es algo normal en cualquier escuela del mundo, del país y de la localidad.

\section{Metodología}

La investigación es soportada desde el paradigma histórico hermenéutico, de tipo cualitativa, descriptiva, por lo que permite identificar características del objeto de estudio, señala formas de conducta y actitudes del universo investigado, además establece una serie de comportamientos concretos que descubre y comprueba la asociación entre categorías de investigación. De acuerdo con los propósitos planteados, el investigador señala el tipo de descripción que se propone realizar (Martínez 2006).

\section{Escenarios y actores}

El desarrollo del estudio se llevó a cabo en la Institución Educativa Santa Cruz de Bálsamo, corregimiento del Municipio de Concordia en el Departamento del Magdalena. Para la aplicación de los instrumentos participaron (51) estudiantes de los grados $4^{\circ}$ y $5^{\circ}$ con una distribución de 25 estudiantes de cada curso. 
Técnicas e instrumentos de recolección de la información

Para la recopilación de la información se utilizó la técnica de entrevista estructurada, la cual pasó por varios filtros, entre ellos la redacción y selección de preguntas, hasta la validación de la misma. Otras de las técnicas empleadas fue la observación directa, y el registro se llevó a cabo en un diario de campo.

\section{Procedimiento}

Momento (1): Se seleccionan los instrumentos a utilizar.

Momento (2): Se especifican las categorías de estudio.

Momento (3): Se escoge los instrumentos de recolección de información.

Momento (4): Con base a las categorías de estudio: Convivencia, estrategia pe- dagógica; se aplicó a los participantes la entrevista, la cual pretende describir el fenómeno que se estudia partiendo del nivel de la investigación (no experimental) en donde se observa, y analiza tal y como acontece.

Momento (5): Una vez se tiene los resultados de la entrevista estructurada y la observación registrada se interpretan y comprenden los resultados a la luz de lo que exponen los teóricos que soportan la investigación.

Momento (6): Se relacionan los resultados y se muestran los hallazgos.

\section{Resultados}

A continuación, se presentan los resultados, de acuerdo a los instrumentos que se les aplicaron a los estudiantes de los grados $4^{\circ}$ y $5^{\circ}$ de la IED Santa Cruz de Bálsamo.

\begin{tabular}{|c|c|c|c|}
\hline $\begin{array}{l}\text { Categoría de } \\
\text { estudio }\end{array}$ & $\begin{array}{c}\text { Pregunta } \\
\text { orientadora }\end{array}$ & $\begin{array}{l}\text { Discurso de } \\
\text { los actores }\end{array}$ & $\begin{array}{c}\text { Articulación y } \\
\text { sistematización teórica }\end{array}$ \\
\hline Convivencia & $\begin{array}{l}\text { ¿Cuál cree usted } \\
\text { que son los } \\
\text { problemas más } \\
\text { frecuentes que } \\
\text { se presentan } \\
\text { en el salón de } \\
\text { clases y por qué? }\end{array}$ & $\begin{array}{l}\text { "aquellos que se } \\
\text { presentan, los niños } \\
\text { peleando porque } \\
\text { se roban las cosas, } \\
\text { entonces salen de pelea", } \\
\text { "el desorden, el robo, el } \\
\text { bullying, porque a los } \\
\text { compañeros les gusta } \\
\text { mucho hacer esas cosas, } \\
\text { sobre todo el bullying, } \\
\text { ellos no quieren cambiar } \\
\text { i respetar", "se pierden } \\
\text { los lapiceros y hacen } \\
\text { desorden en el colegio", } \\
\text { "el robo de lápices, de } \\
\text { libretas, para dañarlas } \\
\text { y no para usarlas", }\end{array}$ & $\begin{array}{l}\text { Son los estudiantes quienes afirman que los problemas } \\
\text { más frecuentes en el aula de clases están asociados a } \\
\text { los robos constantes de los útiles, el comportamiento, } \\
\text { desorden y demás. } \\
\text { Tal como lo expresa Lera. (2003). El papel del conflicto } \\
\text { en las relaciones no puede ser ignorado. En un grupo de } \\
\text { personas que comparten unas actividades y un contexto } \\
\text { lo esperado es que surjan conflictos de intereses, malos } \\
\text { entendidos y problemas y es en el aula de clases, en } \\
\text { donde se observa bullying, comportamientos inadecuados } \\
\text { evidenciados en desorden, agresiones, maltrato verbal y en } \\
\text { los casos extremos incurren al físico. } \\
\text { En concordancia con lo anterior, se infiere que es en la } \\
\text { escuela, en donde se desarrollan agresiones continuadas } \\
\text { de carácter variado en las que hay un desequilibrio de } \\
\text { poder entre víctima y agresor; no necesariamente tiene } \\
\text { que existir violencia, y puede haber desde la agresión } \\
\text { física hasta la psicológica. Estos casos de discriminación } \\
\text { suelen ser más abundantes en la etapa de Educación } \\
\text { Secundaria Obligatoria, y no solamente afecta al } \\
\text { alumnado, sino que también el propio profesorado sufre } \\
\text { dichas agresiones (Hurtado, 2008). }\end{array}$ \\
\hline
\end{tabular}




\begin{tabular}{|c|c|c|}
\hline $\begin{array}{c}\text { Categoría de } \\
\text { estudio }\end{array}$ & $\begin{array}{c}\text { Pregunta } \\
\text { orientadora }\end{array}$ & $\begin{array}{l}\text { Discurso de } \\
\text { los actores }\end{array}$ \\
\hline Convivencia & $\begin{array}{l}\text { ¿Cuál cree usted } \\
\text { que son los } \\
\text { problemas más } \\
\text { frecuentes que } \\
\text { se presentan } \\
\text { en el salón de } \\
\text { clases y por qué? }\end{array}$ & $\begin{array}{l}\text { "Los compañeros se } \\
\text { salen de clases, no } \\
\text { respetan, se roban los } \\
\text { útiles", "hacen mucho } \\
\text { desorden, no le hacen } \\
\text { caso a los profesores", } \\
\text { "intolerancia, irrespeto, } \\
\text { robo, ninguno respeta al } \\
\text { profesor", "el desorden, } \\
\text { la desobediencia", } \\
\text { "pelear con los } \\
\text { compañeros, porque } \\
\text { se colocan apodos y } \\
\text { no respetan”, maltrato } \\
\text { y agresiones", pelea } \\
\text { constante por el robo de } \\
\text { lápices", "el robo, las } \\
\text { maldades y ofensas", } \\
\text { "hablan mucho en clases } \\
\text { y no prestan atención". }\end{array}$ \\
\hline
\end{tabular}

Convivencia

¿Cuándo tienes problemas en casa, lo reflejas agrediendo a tus compañeros?

"No porque es mala
influencia para los
más pequeños", "no,
porque es malo pelear",
"no porque ellos no
tienen la culpa de lo
que a mí me pasa en
mi casa", "no porque
no me gusta maltratar
a mis compañeros",
"no, porque yo no tengo
problemas en mi casa",
"no, porque eso es con
nuestros papas y no se lo
digo a mis compañeros",
"no porque a mí no me
gusta pelear con mis
compañeros por cosas
personales", "no, los
resuelvo en casa con mi
familia”, "no, mejor me
voy para la calle, porque
no sigo peleando", "no,
yo solo coloco cara, y no
les prestó atención, para
que no sepan lo que me
pasa".

"No porque es mala influencia para los más pequeños”, "no, porque es malo pelear", "no porque ellos no tienen la culpa de lo que a mi me pasa en a mis compañeros", "no, porque yo no tengo problemas en mi casa", "no, porque eso es con nuestros papas y no se lo digo a mis compañeros", gusta pelear con mis compañeros por cosas personales", "no, los resuelvo en casa con $m$ familia", "no, mejor me voy para la calle, porque no sigo peleando", "no, yo solo coloco cara, y no pasa".
Articulación y

sistematización teórica

Por otro lado, dos estudiantes asocian como problemas el siguiente aspecto: el problema es que "partieron la puerta".

Para lo anteriormente expuesto, Baroli, (2008), citando a Trianes (2000) lo asocian a una categoría que afecta la convivencia escolar, la indisciplina; que consiste en "el desacato al reglamento de organización y funcionamiento que se produce cotidianamente". Ésta más que entorpecer la convivencia con el compañero, buscan realizar actividades que promuevan el desorden y desautoricen el orden y control dentro de la institución educativa. Finalmente, un estudiante asume que uno de los inconvenientes más frecuentes en aula de clases se traslada los ambientes educativos de aprendizajes tal como lo expone: el problema es que hace mucho calor en el salón. Lo anterior contribuye a lo que Vásquez (2010), citando a Higgins (s.f) afirma cuando dicen que "ambientes de aprendizaje extremadamente pobres tienen un efecto negativo en los estudiantes y el personal docente, así como que, al mejorarlo, se obtienen beneficios significativos".

La calidad interna del aire, ventilación y confort térmico, iluminación, acústica y el tamaño de la escuela y de las aulas son algunos de los atributos espaciales que pueden incidir sobre el rendimiento de alumnos y profesores.

Indiscutible que los conflictos entre padres e hijos aumentan en la adolescencia, y que éstos se han considerado como un indicador de disfunción familiar. Oliva. (2006). Da una opinión teniendo en cuenta lo que expresaron los estudiantes, e infiere que la estrategia seguida para la resolución del conflicto tiene en cuenta los cambios que se viven en la adolescencia, siendo común que las primeras alternativas para la solución de problemas no sean la negociación, por el contrario, se dé el aislamiento. Por el contrario, muchas veces se muestren en el colegio con conductas distintas a las normalmente expresadas, (aislado, agresivo y con apatía académica).

En la Relaciones familiares y desarrollo adolescente medida en que vayan pasando los años la sumisión irá disminuyendo mientras que aumentarán la retirada y la negociación.

Por otro lado, los hallazgos encontrados permiten dar cuenta de la participación que ejercen los "otros" en los problemas familiares, tal como lo expuso un estudiante a continuación: "cuando hay problemas e ni casa lo refiero a mis vecinos para que ayuden".

Tal como lo expresa (Herriko, s.f). La participación de las personas en las relaciones, está marcado por lo que se conoce como influencia, pero esta influencia se lleva a cabo primero, interiormente, a partir de lo que el sujeto constantemente valora frente a sus actos. Es por esto resulta indispensable tomar en cuenta el apoyo que nos brindan las personas cercanas ante la presentación de conflictos. 


\begin{tabular}{|c|c|c|c|}
\hline $\begin{array}{c}\text { Categoría de } \\
\text { estudio }\end{array}$ & $\begin{array}{c}\text { Pregunta } \\
\text { orientadora }\end{array}$ & $\begin{array}{l}\text { Discurso de } \\
\text { los actores }\end{array}$ & $\begin{array}{c}\text { Articulación y } \\
\text { sistematización teórica }\end{array}$ \\
\hline Convivencia & $\begin{array}{l}\text { ¿Cuáles son los } \\
\text { problemas más } \\
\text { frecuentes en tu } \\
\text { familia y como } \\
\text { lo solucionan? }\end{array}$ & $\begin{array}{l}\text { "Cuando se discute, pero } \\
\text { se arregla dialogando } \\
\text { con la familia, y } \\
\text { nos sirve un poco } \\
\text { para solucionarlos", } \\
\text { "cuando se discute por } \\
\text { cualquier bobada, los } \\
\text { resolvemos preguntando } \\
\text { y dialogando", "las } \\
\text { discusiones con amor, } \\
\text { y alguien se equivoca } \\
\text { y ahi comenzamos } \\
\text { a discutir", "que no } \\
\text { quieren hacer un } \\
\text { mandado, luego se } \\
\text { soluciona hablando", } \\
\text { "las constantes } \\
\text { discusiones, pero se } \\
\text { solucionan hablando". }\end{array}$ & $\begin{array}{l}\text { Los hallazgos anteriores permiten informar que los } \\
\text { adolescentes reconocen que existen problemas constantes, } \\
\text { que pueden ir desde los más sencillos, hasta los más } \\
\text { complejos, pero que siempre buscan alternativas de } \\
\text { solución. Las relaciones familiares durante la adolescencia } \\
\text { se han convertido en uno de los tópicos que suscitan } \\
\text { más interés entre investigadores está el deterioro de las } \\
\text { relaciones familiares con la llegada de la pubertad, sin } \\
\text { embargo se resalta que a pesar de las etapas por las que } \\
\text { esté pasando el adolescente y los conflictos familiares que } \\
\text { dentro de esta se puedan ocasionar, los lazos afectivos se } \\
\text { mantienen (Oliva, 2006). }\end{array}$ \\
\hline $\begin{array}{l}\text { Estrategia } \\
\text { pedagógica }\end{array}$ & $\begin{array}{l}\text { ¿Cuándo tienes } \\
\text { una situación de } \\
\text { irrespeto con los } \\
\text { docentes como } \\
\text { lo solucionas } \\
\text { con él? ¿Qué } \\
\text { estrategia } \\
\text { utiliza el } \\
\text { docente para } \\
\text { mediar la } \\
\text { situación? }\end{array}$ & $\begin{array}{l}\text { "Dialogando con el } \\
\text { docente para que } \\
\text { para que ayude a los } \\
\text { demás estudiantes con } \\
\text { las tareas, "cuando } \\
\text { hay una situación de } \\
\text { irrespeto se dialoga } \\
\text { con el docente, para } \\
\text { buscar la solución”, } \\
\text { "dialogando con el } \\
\text { docente, explicando } \\
\text { los problemas que } \\
\text { tengo", "hablando con } \\
\text { el estudiante, para que } \\
\text { reflexione”, "se solicita, } \\
\text { pidiéndole perdón a } \\
\text { ellos, haciendo un alto } \\
\text { y recibiendo consejos", } \\
\text { "lo solucionamos } \\
\text { pidiéndonos disculpas } \\
\text { y hablando del } \\
\text { problema”, "pidiéndole } \\
\text { perdón, y diciéndole } \\
\text { que cumpliré con mis } \\
\text { obligaciones, también } \\
\text { el exijo respeto de su } \\
\text { parte”, nos disculpamos } \\
\text { y nos damos la mano”, } \\
\text { "yo le pediría perdón”, } \\
\text { "le pido perdón y le digo } \\
\text { que no peleemos más”, } \\
\text { "le explico la situación } \\
\text { y así se arreglan los } \\
\text { irrespetos”. }\end{array}$ & $\begin{array}{l}\text { Teniendo en cuenta lo expuesto por los estudiantes, se } \\
\text { dice que son ellos los que sí reconocen que existen distinto } \\
\text { tipo de inconvenientes en la relación estudiante-docente } \\
\text { y que es necesario buscar alternativas de solución que } \\
\text { contribuya a una sana convivencia, basado en el respeto y } \\
\text { la empatía. } \\
\text { Según Salas (2012), Un buen educador diseña, crea e } \\
\text { implementa constantemente mecanismos encaminados } \\
\text { a garantizar la comunicación fluida con sus estudiantes; } \\
\text { de tal forma que se promueva el interés por aprender } \\
\text { a aprender. Para esta autora el docente debe dirigir, } \\
\text { inequívocamente todo su esfuerzo y dedicación a } \\
\text { captar la atención de los aprendizajes priorizando la } \\
\text { comunicación, y por su parte el estudiante, también } \\
\text { debe tomar iniciativas que promuevan la participación } \\
\text { basada en el respeto por el otro, el acato de normas, y la } \\
\text { responsabilidad de ciertas tareas. } \\
\text { Por otra parte, un estudiante responde: "firmando un acta } \\
\text { se soluciona el problema". } \\
\text { No desconoce los problemas que se puede presentar, } \\
\text { sin embargo, sabe que existen lineamientos, normas y } \\
\text { compromisos que cumplir, y que de violentaras, trae sus } \\
\text { consecuencias. Los maestros, por su lado deberán crear } \\
\text { espacios de socialización frente a situaciones presentadas } \\
\text { en el aula de clases, y brindar estrategias para su } \\
\text { solución, y dependiendo de la gravedad de los casos seguir } \\
\text { los conductos y participar a las instancias pertinentes. }\end{array}$ \\
\hline
\end{tabular}




\begin{tabular}{|c|c|c|c|}
\hline $\begin{array}{c}\text { Categoría de } \\
\text { estudio }\end{array}$ & $\begin{array}{l}\text { Pregunta } \\
\text { orientadora }\end{array}$ & $\begin{array}{l}\text { Discurso de } \\
\text { los actores }\end{array}$ & $\begin{array}{c}\text { Articulación y } \\
\text { sistematización teórica }\end{array}$ \\
\hline $\begin{array}{l}\text { Estrategia } \\
\text { pedagógica }\end{array}$ & $\begin{array}{l}\text { ¿La institución } \\
\text { educativa se } \\
\text { preocupa por } \\
\text { programas que } \\
\text { fortalezcan } \\
\text { las relaciones } \\
\text { interpersonales } \\
\text { y la mejora } \\
\text { de una sana } \\
\text { convivencia? }\end{array}$ & $\begin{array}{l}\text { "Si, a cada rato están } \\
\text { haciendo actividades, } \\
\text { ahora con el uso de } \\
\text { las tecnologías nos } \\
\text { hacen bastantes } \\
\text { actividades", "si, el } \\
\text { colegio se preocupa } \\
\text { por la convivencia”, } \\
\text { "si hace actividades" } \\
\text { "si”, "cuando peleamos } \\
\text { los profesores hacen } \\
\text { actividades y llaman a } \\
\text { los papás de uno", "si, } \\
\text { hacemos dramatizados", } \\
\text { "si, en el salón los } \\
\text { profesores nos viven } \\
\text { diciendo que no } \\
\text { peleemos más, y cuando } \\
\text { lo hacemos nos obligan } \\
\text { a pedirnos disculpas". }\end{array}$ & $\begin{array}{l}\text { Tal como lo expresa Meyer (1984), el compromiso de } \\
\text { las instituciones educativas, es generar estrategias de } \\
\text { apoyo para el desarrollo de competencias y habilidades, } \\
\text { apoyados en las herramientas tecnológicas, desde el uso } \\
\text { pedagógico, las cuales promueven en el estudiante a } \\
\text { afianzar sus competencias, logrando ser asertivo en la } \\
\text { solución de los problemas que se le vayan presentando. } \\
\text { motivan a Y solo un estudiante afirmó que el colegio "no } \\
\text { hacen ningún programa". A lo que es contradictorio con lo } \\
\text { que Tafur } 2016 \text { expone. "toda institución educativa debe } \\
\text { propiciar espacios que fundamente la sana convivencia, } \\
\text { y todo maestro debe articular en su práctica pedagógica, } \\
\text { actividades que logren ambientes armoniosos de formación } \\
\text { académica y humana. }\end{array}$ \\
\hline
\end{tabular}

Fuente: Elaboración propia. (2018).

\section{Conclusiones}

Se pudo distinguir que uno de los mayores problemas que se presentan en la Institución Educativa, está asociado directamente al robo de útiles escolares, agresión, y bullying. Los estudiantes emplean pocos canales de comunicación asertivos para solucionar cualquier tipo de problemas, por el contrario, imitan conductas que le permitan sobresalir ante distintas situaciones, empleando la agresividad como medio para lograr sus objetivos.

En cuanto a su relación con los docentes, tienden a respetar la figura de autoridad en el aula de clases, pero cuando no están de acuerdo con ciertas situaciones en muchas ocasiones pueden evidenciar actitudes groseras y no cumplir con los compromisos asignados.

Los padres son un modelo a seguir al interior de las familias, es por ello que los comportamientos y relaciones que se impartan al interior del hogar serán ejemplo para manifestarlas en el aula de clases. No obstante; tienen situaciones que generan conflicto con sus hijos ya sea por irresponsabilidades familiares y escolares, rebeldía, irrespeto entre otros. En muchas ocasiones aplican el castigo como alternativa de solución, en otras ocasiones el maltrato físico y verbal, y también utilizan el dialogo para concertar.

Los estudiantes reconocen la necesidad de tener relaciones, e identifican en sus compañeros de clases, la consecución de lazos muy fuertes, en donde se apoyan, comparten sentimientos, pensamientos e ideas, pero, también se generan conflictos, discusiones, maltratos por situaciones diversas. Describen la importancia de crear buenas relaciones interpersonales, que sirvan para ayudarse ante las diferentes necesidades que se puedan presentar, como así mismo buscan relaciones y aprovechar las competencias y capacidades de las personas con las que se rodean para aprender de cada experiencia. 
Finalmente, La institución educativa ha venido trabajando en la participación trimodal estudiante- docentes- padres de familia, con la finalidad que sean los padres los que también se involucren en las actividades académicas, y logren integrar experiencias, aprender al igual que los estudiantes nuevos manejos y estrategias que contribuyan a la convivencia sana en todos los escenarios en los que se desenvuelven los hijos.

\section{Referencias}

Alvarez, S. (2011). Estrategias de comunicación para padres con hijos adolescentes. [Tesis de Maestría]. Facultad de Educación. Mérida. Recuperado de http://www.culturadelalegalidad.org. $\mathrm{mx} /$ recursos/Contenidos/Familia/documentos/Estrategias\%20de\%20comunicacion $\% 20$ para $\% 20$ padres $\% 20$ con $\% 20$ hijos\%20adolescentes.pdf

Avendaño, I., Cortés O. y Guerrero, H. (2015). Competencias sociales y tecnologías de la información y la comunicación como factores asociados al desempeño en estudiantes de básica primaria con experiencia de desplazamiento forzado Diversitas: Perspectivas en Psicología, 11(1). 13-36. Recuperado de http://www. scielo.org.co/pdf/dpp/v11n1/v11n1a02. pdf

Betancur, S., Carmona, L., Contreras, R., Karam, J., Maestre, N., Romero, Y. y Uribe, S. (2014). Videojuegos y tic como Estrategias Pedagógicas: Formación para el uso seguro de internet. Cultura Educación y Sociedad, 5(1). Recuperado de https://revistascientificas.cuc.edu. co/culturaeducacionysociedad/article/ view/997

Baroli, A. (s.f.). Identificación de problemas institucionales. Recuperado de http:// www.igualdadycalidadcba.gov.ar/SIPEC-CBA/publicaciones/documentos/ Problemasinstitucionales.pdf
Casadiego, J., Martínez, C., Riatiga, A. y Lopez, E. (2015). Habilidades de comunicación asertiva como estrategia en la resolución de conflictos familiares que permite contribuir al desarrollo humano integral en la familia. [Tesis de Pregrado]. Universidad nacional, abierta y a distancia, UNAD.

Clemente, R. (2015). Relaciones interpersonales y desarrollo humano. Castellón de la plana: Universitat Jaume I. http:// dx.doi.org/10.6035/Llico.2015.2016

Delgado, R. (2010). Relaciones interpersonales en la adolescencia: Implementación de un programa de entrenamiento en asertividad y habilidades sociales para adolescentes de $1^{o}$ y $2^{\circ}$ de la ESO. [Tesis de Pregrado]. Universidad de Granada, Granada.

González, J., Moreno, L. y Rodríguez, M. (2015). La integración y sus convenios internacionales en América Latina. Económicas CUC, 36(2), 79-94.

Guerrero, H. y Cepeda, M. (2016). Uso de estrategias pedagógicas para el fortalecimiento de la convivencia escolar de jóvenes vulnerables. Revista de Pedagogía, 37(101). 57-79 Recuperado de http://saber. ucv.ve/ojs/index.php/rev_ped/article/ view/12501.

Hurtado, J. (2008). Gestión de instituciones escolares: La cultura escolar Chilena y la influencia de las variables del soft management en el rendimiento académico. [Tesis de Maestría]. Pontificia Universidad Católica de Chile, Santiago de Chile. Recuperado de https://repositorio.uc.cl/bitstream/handle/11534/1434/505062. pdf?sequence=

Kadi, O. y Acevedo, Á. (2014). Liderazgo ético frente a la diversidad cultural dentro de las organizaciones con régimen disciplinario. Económicas CUC, 35(2), 75-88.

Lera, M. (2003). Las relaciones personales en los centros educativos. Sevilla: Universidad de Sevilla. 
Llorent, V. y Lopez, A. (2012). Estudio de las relaciones interpersonales en aulas con alumnos "diferentes". Campo abierto, 31(2). 87-109.

Lopez, V. (2014). Convivencia Escolar. Apuntes. 4. 1-15. Recuperado de http:// www.unesco.org/new/fileadmin/MULTIMEDIA/FIELD/Santiago/pdf/ APUNTE04-ESP.pdf

Mujica, C. (2013). Propuesta de solución de conflictos entre padres e hijos adolescentes. Revista vinculando. Recuperado de http://vinculando.org/padres_e_hijos_familia/solucion-de-conflictos-quevivencian-los-padres-con-sus-hijos-adolescentes.html

Oliva, A. (2006). Relaciones familiares y desarrollo adolescente. Anuario de Psicología. 37(3). 37(3). 209-223 Recuperado de http://www.raco.cat/index.php/ anuariopsicologia/article/view File/61838/82584\&q=chicas

Pantoja, A. (2005). La gestión de conflictos en el aula. Factores determinantes y propuestas de intervención. Madrid: Ministerio de Educación y Ciencia. Recuperado de https://www4.ujaen.es/ apantoja/mis _libros/gestion_confli_05.pdf

República de Colombia. Ministerio de Educación Nacional. (2012). Una propuesta pedagógica para la educación de la primera infancia. Recuperado de http:// www.colombiaaprende.edu.co/html/familia/1597/articles-310603_docu2.pdf

República de Colombia. Ministerio de Educación Nacional. (2017). Encuesta nacional de deserción escolar. ENDE. Recuperado de http://www.mineducacion.gov.co/1621/articles-293664_archivo_pdf_resultados_ETC.pdf
Romero, G., Suárez, R. y Rodríguez, H. (2018). Modelo de capacidades de innovación para instituciones de educación superior. INGE CUC, 14(1), 87-100. https:// doi.org/10.17981/ingecuc.14.1.2018.8

Sanchez, F. (2017). Conflictos entre padres e hijos: el problema de la disciplina. Adolescere, 5(1). 70-80. Universidad de Salamanca. Colombia.

Sanchez, J. (2015). El adolescente y su familia. Diagnóstico. 53(3). Recuperado de http://www.fihu-diagnostico.org.pe/wpcontent/uploads/2016/09/05-simposiomedicina-del-adolescente-el-adolescentey-su-familia-dr-juan-carlos-lengua-sanchez-final-pdf.pdf

Suárez, O. (2016). Aproximación al origen de la noción de objeto de aprendizaje: revisión histórico-bibliográfica. INGE CUC, 12(2), 26-40. https://doi.org/10.17981/ingecuc.12.2.2016.03

UNESCO. (2011). Clima, conflictos y violencia en la escuela. Argentina: Flasco. Recuperado de https://www.unicef.org/ argentina/spanish/clima_conflicto_violencia_escuelas.pdf

Valdarrosa, A. (s.f). Guía familiar solución de problemas en la adolescencia. Recuperado de http://www.edu.xunta.gal/centros/iespontecaldelas/system/files/ guia\%20anpa\%20resolucion\%20de\%20 conflictos\%20con\%20ilustraciones.pdf

Vásquez, M. (2010). Condiciones ambientales en la escuela. Recuperado de http:// www.consumer.es/web/es/educacion/escolar/2010/10/22/196660.php

Velazco, M. y Mosquera, F. (2010). Manual de Estrategias didácticas. Recuperado de http://comisioniberoamericana.org/ gallery/manual-estrategias-didacticas. pdf 